\title{
Achievements in cancer research supported by National Natural Science Foundation of China
}

\author{
HONG Wei, LI Cui, ZHANG LiPing, XU YanYing, ZHANG Jun, HU Hai \& DONG ErDan* \\ Department of Health Sciences, National Natural Science Foundation of China, Beijing 100085, China
}

Received November 19, 2012; accepted November 30, 2012

For the past two decades, great achievements have been made in cancer research of China and the majority of them were supported by the National Natural Science Foundation of China (NSFC). In this article, we reviewed the funding history of NSFC including the number of proposals, the amount of grants, distribution of supported program categories during the period of 1987 to 2012 and the representative achievements made by continuous support of NSFC. Also, the impacts of NSFC support on discipline development and talent fostering were analyzed. From 1987 to 2012, a total of 8738 proposals related to cancer research were funded by NSFC. The percentages of the Research Program, Talent-Training Program, and the Research Environment Program were $61.19 \%, 36.32 \%$, and $2.49 \%$, respectively. Under the support of NSFC, cancer research in China has achieved a significantly rapid and balanced development in both disciplines and scientist resources.

cancer research, National Natural Science Foundation of China, achievements

Citation: Hong W, Li C, Zhang L P, et al. Achievements in cancer research supported by National Natural Science Foundation of China. Chin Sci Bull, 2013, 58: 39-43, doi: $10.1007 / \mathrm{s} 11434-012-5633-x$

National Natural Science Foundation of China (NSFC) has held on its policies to constantly support basic and applied basic research since its establishment in 1986. Programs in cancer research are now administrated by Division of Oncology at Department of Health Sciences of NSFC. The scopes of grant support in Division of Oncology cover (1) cancer biology, including etiology, carcinogenesis, genetics, immunology, prevention, recurrence and metastasis of tumors, and cancer stem cells; (2) cancer clinics, including diagnosis, chemotherapy, radiotherapy, biotherapy, integrated therapeutics, and rehabilitation (social and psychological rehabilitation); and (3) new techniques in cancer research. On the other hand, the discipline in Division of Oncology is also classified based on different human medical systems/organs. Overall the scopes of application codes cover nearly every aspect of cancer research with the exception of tumor epidemiology and leukemia, which are managed by Division of Preventive Medicine and Division of Haematologic and Lymphatic System Diseases, respectively.

*Corresponding author (email: donged@nsfc.gov.cn)

\section{Overview of NSFC grant support on cancer research}

NSFC has 3 programs integrated in the whole funding system with different focuses, which are designated for Research, Talent-Training, and Research Environment programs, respectively. From 1987 to 2012, NSFC has funded 8738 programs in cancer research with a total sum of approximately 3 billion RMB. The number and budget of the funded proposals in these 3 programs over the period are shown in Table 1. Among the funded proposals, $61.19 \%$ were supported via Research Program, $36.32 \%$ via TalentTraining Program and $2.49 \%$ via Research Environment Program. There are also different subcategories of programs corresponding to each one of the above, and the number and size in oncology research for each category supported by NSFC from 1987 to 2012 are shown in Table 2. Importantly, while the total funding of NSFC has been improved gradually, the budget of NSFC and, particularly, the support for cancer research have been increased dramatically during the 
Table 1 Application and funding status in oncology of NSFC in China, 1987-2012

\begin{tabular}{lccc}
\hline \multicolumn{1}{c}{ Program } & Applicant no. & Funding no. & Funding size (million RMB) \\
\hline Research Program & 31515 & 5347 & 2085.547 \\
Talent-Training Program & 16839 & 3174 & 813.391 \\
Research Environment Program & 493 & 217 & 34.896 \\
Total & 48847 & 8738 & 2933.834 \\
\hline
\end{tabular}

Table 2 Funding categories in oncology supported by NSFC in China, 1987-2012

\begin{tabular}{|c|c|c|c|}
\hline Program & Funding subcategories & Funding no. & $\begin{array}{l}\text { Funding size } \\
\text { (million RMB) }\end{array}$ \\
\hline \multirow{6}{*}{ Research Program } & General Program & 4848 & 1687.060 \\
\hline & Key Program & 96 & 190.640 \\
\hline & Major Program & 11 & 30.660 \\
\hline & Major Research Plan & 119 & 118.700 \\
\hline & Major International Cooperation & 53 & 35.117 \\
\hline & The President's and Department Directors' Funds & 220 & 23.370 \\
\hline \multirow{7}{*}{ Talent-Training Program } & National Science Fund for Distinguished Young Scholars (DYS) & 60 & 80.300 \\
\hline & Outstanding Young Investigator Fund & 9 & 9.000 \\
\hline & Young Scientists Fund & 2619 & 533.770 \\
\hline & Fund for Less Developed Regions & 446 & 146.521 \\
\hline & Science Fund for Creative Research Groups & 7 & 32.800 \\
\hline & Joint Research Fund for Overseas Chinese Scholars in Hong Kong and Macao & 11 & 2.200 \\
\hline & Joint Research Projects for Oversea Young Scientists & 22 & 8.800 \\
\hline \multirow{3}{*}{ Research Environment Program } & Special Fund for Basic Research on Scientific Instruments and Journals & 156 & 16.171 \\
\hline & Programs of Joint Funds & 16 & 17.200 \\
\hline & $\begin{array}{l}\text { International (Regional) Cooperation and Exchange } \\
\text { Hold International Academic Workshops in China }\end{array}$ & 45 & 1.525 \\
\hline Total & & 8738 & 2933.834 \\
\hline
\end{tabular}

past two years (Figure 1).

\section{Breakthroughs in cancer research supported by NSFC}

A series of groundbreaking achievements have been made

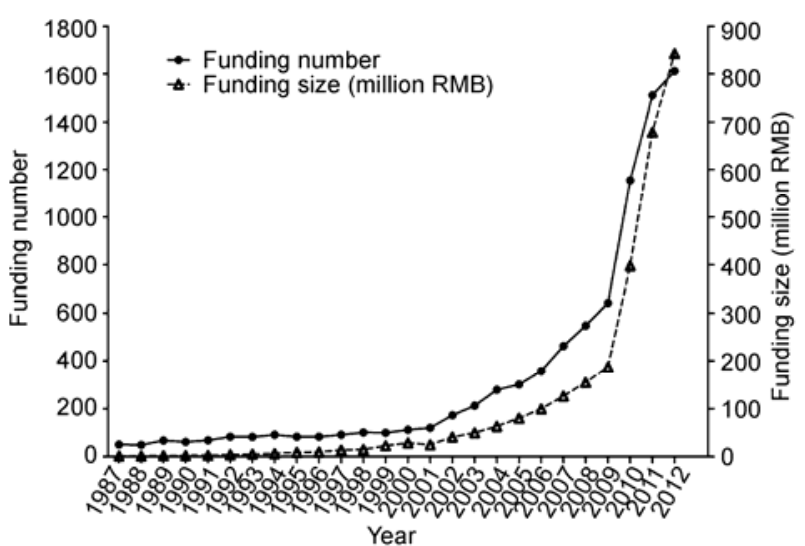

Figure 1 Growth of funding number and size in oncology supported by NSFC in China, 1987-2012. in cancer research under NSFC support, particularly, in the fields of cancer genetics and epigenetics, signal transduction, microRNA and tumor stem cells, tumor microenvironment, inflammation and tumor metastasis, tumor immunology, and prevention and treatment of cancer during the past 26 years.

(i) Cancer genetics and epigenetics. Shang YongFeng's group identified a couple of oncogenes co-regulated by estrogen and tamoxifen. One of the genes, $P A X 2$, regulates the proliferation of endometrial cancer. They found that estrogen and tamoxifen promote the growth of endometrium cancers by upregulating $P A X 2$ expression via relieving hypermethylation at the Cpg islands of its promoter region, which is normally observed in normal endometrial cells [1]. Due to his novel findings in this field, Shang was invited to contribute a review on the molecular mechanisms of endometrial cancer induced by estrogen and tamoxifen [2]. They also found that lysine-specific demethylase 1 (LSD1) is an integral component of the $\mathrm{Mi}-2 /$ nucleosome remodeling and deacetylase (NuRD) complex, which regulates several cellular signaling pathways including the TGF- $\beta 1$ signaling pathway that are critically involved in cell proliferation, survival, and epithelial-to-mesenchymal transition (EMT). 
Moreover, they found that LSD1 is down-regulated in breast carcinomas and its level of expression is negatively correlated with that of TGF- $\beta 1$. Their data provided a molecular basis for the interplay of histone demethylation and deacetylation in chromatin remodeling [3]. In addition, another research group led by Zhan QiMin identified NLP gene and demonstrated that it is associated with the onset of breast cancers in a transgenic mouse model [4].

In search of tumor susceptibility genes, breakthroughs have been made in nasopharyngeal carcinoma (NPC), esophagus and liver cancers, which have obvious topological distribution and are highly incident in China. Zeng YiXin's group discovered the mode of inheritance of NPC in Guangdong families and identified a susceptibility locus on 4 section 2 to 5 in region 1 of the short arm of chromosome 4 [5], and recently, they also revealed 3 new risk loci of NPC on chromosomes 3, 9 and 13, and confirmed the known locus on chromosome 6, using a genome-wide association study with large scale of NPC cases and controls [6]. Lin DongXin's team identified several new and functional variations that are associated with susceptibility to multiple cancer including lung, esophagus, and gastric cancer [7-9]. They revealed 3 new susceptibility loci for esophageal squamous-cell carcinoma and identified 5 new susceptibility loci for pancreatic cancer in Chinese populations by genome-wide association study [10].

(ii) Signal transduction in cancer. Wang HongYang's group showed that the expression of p28GANK may serve as a molecular marker for diagnosis of liver cancer, and that p28GANK may tip the balance of proliferation and apoptosis of hepatoma cell by regulating the phosphorylation and expressive level of tumor suppressors, including RB1. Moreover, they found that the alpha subunit of ciliary's neurotrophic factor receptor can activate both PI3K/Akt and MAPK signal transduction pathways upon engagement by ciliary's neurotrophic factor, which contributes to the glycometabolism process of normal hepatocytes and hepatocarcinoma cells [11,12]. In the field of breast cancers, Zhang XueMin's team made remarkable progress to elucidate the mechanism of tamoxifen-resistance of breast cancer. They identified a new protein, CUE-domain-containing protein (CUEDC2), which reduces sumoylation of progesterone receptor. Simultaneously, CUEDC2 serves as an adaptor protein to deactivate IKK by recruiting PP1 to the complex and thus targets IKK for dephosphorylation and inactivation, which contributes to the resistance of breast cancer cells to tamoxifen. They also found that overexpression of CUEDC2 is associated with poor outcome of breast cancer patients and may thus serve as a potential new target to reverse the resistance to tamoxifen. Further study revealed that CUEDC2 is a key regulator of mitosis progression, and its dysregulation may favor tumor development due to chromosomal instability [13-16].

(iii) Tumor related miRNAs and tumor stem cells. By profiling the microRNAs, Song ErWei's group demonstrat- ed the differential expression of some small non-coding RNAs in breast cancer stem cells, also named breast tumor initiating cells, versus non breast cancer stem cells. More importantly, reduction in let-7 miRNA helps to maintain the self-renewal capacity, undifferentiation state and highly tumorigenic potential of breast cancer stem cells. Ectopic expression of the microRNA retards tumor growth in immunocompromised mice and inhibits breast cancer metastasis. The study proposed an innovative theory that miRNA are crucial to the growth and metastasis of tumors by regulating cancer stem cells, which is a milestone breakthrough in the field of tumor related miRNA and cancer stem cells with citation of nearly 500 times within 5 years [17]. Besides, Wang HongYang's team found that the gene locus harboring $m i R-151$ is amplified at a high frequency in hepatocarcinoma genome leading to high expressive level of $m i R-151$ in hepatocarcinoma. The overexpressed $m i R$ 151 silences a tumor suppressor RhoGDIA and thus promotes metastasis of liver cancers [18].

(iv) Tumor microenvironment, inflammation and tumor metastasis. In studies of tumor microenvironment, Song ErWei's team demonstrated that CCL18 produced by tumor-associated macrophages in breast cancers is associated with metastasis and poor clinical outcome via promoting cancer cell invasion and adhesion. Furthermore, they identified that PITPNM3 is a potential functional receptor for CCL18. The study, published as a featured article in Cancer Cell, was highlighted by Nature. Song ErWei's finding shed new light on the role of chemokines in cancer, and CCL18 may serve as a potential therapeutic targets for breast cancer [19].

After 10 years of hard work, Tang ZhaoYou's team has established a series of models to study metastasis of liver cancers, which include a highly metastatic hepatoma xenografting model inoculated in nude mice, a pair of hepatoma cell lines MHCC97H and MHCC97L with identical cellular genetic background but different metastatic potentials and cell lines HCCLM3 and HCCLM6 with progressively increased metastatic potential. Using the above models, they found that cancer cells with identical genetic background may develop into tumors with differential metastatic potentials, since different tumor microenvironment changes the expression level of oncogenes related to metastasis [20].

(v) Immunology, prevention and treatment of cancer. Scientists in China have made great progress in cancer immunotherapies. For instance, Wei YuQuan's team proposed a new theory in cancer immunology, suggesting that heteroantigens can effectively abolish immune tolerance of malignant tumors, induce anti-cancer immunity, and thus may serve as a novel strategy for cancer immunotherapy [21]. Guo YaJun's team proposed to modulate the antigen presenting function of cancer cells to activate tumor specific immune response triggered by host antigen, suggesting a new strategy to stimulate anti-cancer immunity by fusing the antigen presenting cells with tumor cells [22]. 
Cao XueTao's team systemically conducted basic and translational research on the tumor immunology, including the mechanism of dendritic cells (DC) and their clinical application as immuno-gene therapy. They creatively identified a new cluster of DCs with important immuno-modulation functions and found that matured DCs could further proliferate and differentiate upon stimulation by matrix. New approaches combined with immunotherapy and gene therapy, were applied both in laboratories and clinical studies [23]. Wang ZhiBiao has invented a High Intensity Focused Ultrasound (HIFU) system for cancer treatment, which was the first cancer treatment device with completely independent intellectual property rights in China [24].

\section{Talents fostering under NSFC's support}

NSFC has launched the Talent-Training Program with 7 different subcategories to foster young scientists (Table 2) and has played an important role in scientist training at different levels in China. National Science Fund for Distinguished Young Scholars, considered as a "Top Gun Academy" in fostering young talent scientists in China, supports young scholars who have made outstanding achievements in basic research to aim at their own research directions and to conduct creative studies. The goals of this program are to speed up the independence of young scientific talents, to encourage homecoming of overseas talents and to foster a group of prominent academic pace markers in the leading fronts of world science and technology. In the field of medicine, most of the talented scientists have been supported by National Science Fund for Distinguished Young Scholars before they reach the age of 45 year and some of them have made tremendous progress in oncological research. Of note, these members of the Chinese Academy of Sciences or the Chinese Academy of Engineering, Wang HongYang, Shang YongFeng, Zeng YiXin, He FuChu, Wei YuQuan, Zhang XueMin, Cao XueTao, Fan DaiMin, and Zhan QiMin and Profs. Lin DongXin, Song ErWei, Guo YaJun, Wang ZhiBiao, were all funded by this program and the Key Program of NSFC. Moreover, another Talent-Training Program supporting a group of scientists to perform coordinated research work is also crucial to scientists in cancer research, and the members of the Chinese Academy of Sciences or the Chinese Academy of Engineering Wang HongYang, Cao XueTao, Fan DaiMin and Zhan QiMin are all supported by this program.

Outstanding Young Investigator Fund is another Talent-Training Program freshly established in 2012. As a supplement to the program for Distinguished Young Scholars, this grant serves as an effective bridge to link the existing programs for general Young Scientists Fund and Distinguished Young Scholars, so as to promote the rapid growth of creative scientific talents in China. In 2012, a total of 98 applications were submitted for this funding. Among which,
9 applicants gained support, with a funding rate of $9.18 \%$, reflecting a fierce competition in this field.

In addition to high-impact research papers, the scientific influence of Chinese scientists on cancer research is also demonstrated by their increasing international academic status. More and more outstanding scientists are invited as chairs or keynote speakers in international conferences. For example, Cao XueTao serves as an executive committee member of International Union of Immunological Societies (IUIS), deputy chief-editor of Gene Therapy, co-chief-editor of Cellular and Molecular Immunology, etc. He FuChu serves as a co-principal investigator of the International Human Liver Proteome Project (HLPP), council member of the Human Proteome Organization (HUPO) etc. HLPP is the first human organ-/tissue-based proteome project, and the first high-impact international cooperative program project led by a Chinese scientist, which was reported by $\mathrm{Na}$ ture, Science and Nature Biotechnology. More than 200 manuscripts were published by He's team in the past 10 years in scientific journals including Science, Nature Genetics, Nature Cell Biology, Nature Biotechnology, Nature Methods, Nature Medicine, Nature Protocol and PNAS.

\section{The role of NSFC grant(s) in the national funding structure}

National "973 Program", another national funding source from Ministry of Science and Technology of China, also finances basic cancer research but mainly focuses on major basic scientific questions with limited supporting fields. Hence, "973 Program" places emphasis on only very few research areas, instead of the systematicness of cancer science, and does not carry the mission of discipline development and talent training.

In contrast, NSFC emphasizes systematic, prospective and explorative cancer research fields, with special highlights on building up research foundation and training talented cancer scientists. Hence, the goal of NSFC funding support is to promote balanced development of all research disciplines and to speed up high-impact scientific achievements approaching international standards. Especially, various categories of NSFC programs for scientist training help to expand our talent reserve, to cultivate outstanding young scientists and to build up cooperative research groups for top-level cancer research in China.

$1 \mathrm{Wu} \mathrm{H}$, Chen Y, Liang J, et al. Hypomethylation-linked activation of PAX2 mediates tamoxifen-stimulated endometrial carcinogenesis. Nature, 2005, 438: 981-987

2 Shang Y. Molecular mechanisms of oestrogen and SERMs in endometrial carcinogenesis. Nat Rev Cancer, 2006, 6: 360-368

3 Wang Y, Zhang H, Chen Y, et al. LSD1 is a subunit of the NuRD complex and targets the metastasis programs in breast cancer. Cell, 2009, 138: 660-672

4 Shao S, Liu R, Wang Y, et al. Centrosomal Nlp is an oncogenic pro- 
tein that is gene-amplified in human tumors and causes spontaneous tumorigenesis in transgenic mice. J Clin Invest, 2010, 120: 498-507

5 Feng B, Huang W, Shugart Y, et al. Genome-wide scan for familial nasopharyngeal carcinoma reveals evidence of linkage to chromosome 4. Nat Genet, 2002, 31: 395-399

6 Bei J, Li Y, Jia W, et al. A genome-wide association study of nasopharyngeal carcinoma identifies three new susceptibility loci. Nat Genet, 2010, 42: 599-603

$7 \mathrm{Hu} \mathrm{Z}$, Wu C, Shi Y, et al. A genome-wide association study identifies two new lung cancer susceptibility loci at $13 q 12.12$ and 22 q12.2 in Han Chinese. Nat Genet, 2011, 43: 792-796

8 Wu C, Hu Z, He Z, et al. Genome-wide association study identifies three new susceptibility loci for esophageal squamous-cell carcinoma in Chinese populations. Nat Genet, 2011, 43: 679-684

9 Shi Y, Hu Z, Wu C, et al. A genome-wide association study identifies new susceptibility loci for non-cardia gastric cancer at 3 q13.31 and 5p13.1. Nat Genet, 2011, 43: 1215-1218

10 Wu C, Miao X, Huang L, et al. Genome-wide association study identifies five loci associated with susceptibility to pancreatic cancer in Chinese populations. Nat Genet, 2011, 44: 62-66

$11 \mathrm{Li} \mathrm{H}, \mathrm{Fu} \mathrm{X}$, Chen Y, et al. Use of adenovirus-delivered siRNA to target oncoprotein p28(GANK) in hepatocellular carcinoma. Gastroenterology, 2005, 128: 2029-2041

12 Fu J, Chen Y, Cao J, et al. 1,6p28(GANK) overexpression accelerates hepatocellular carcinoma invasiveness and metastasis via phosphoinositol 3-kinase/AKT/hypoxia-inducible factor-1 alpha pathways. Hepatology, 2011, 53: 181-192

13 Zhang $\mathrm{P}$, Zhao J, Li H, et al. CUE domain containing 2 regulates degradation of progesterone receptor by ubiquitin-proteasome. EMBO J, 2007, 26: 1831-1842

14 Li H, Liu H, Wang C, et al. Deactivation of the kinase IKK by
CUEDC2 through recruitment of the phosphatase PP1. Nat Immunol, 2008, 9: 533-541

15 Pan X, Zhou T, Tai Y H, et al. Elevated expression of CUEDC2 protein confers endocrine resistance in breast cancer. Nat Med, 2011, 17: 708-714

16 Gao Y, Li T, Chang Y, et al. Cdk1-phosphorylated CUEDC2 promotes spindle checkpoint inactivation and chromosomal instability. Nat Cell Biol, 2011,13: 924-933

17 Yu F, Yao H, Zhu P, et al. let-7 regulates self renewal and tumorigenicity of breast cancer cells. Cell, 2007, 131: 1109-1123

18 Ding J, Huang S, Wu S, et al. Gain of miR-151 on chromosome 8q24.3 facilitates tumour cell migration and spreading through downregulating RhoGDIA. Nat Cell Biol, 2010, 12: 390-399

19 Chen J, YaoY, Gong C, et al. CCL18 from tumor-associated macrophages promotes breast cancer metastasis via PITPNM3. Cancer Cell, 2011, 19: 541-555

20 Ye Q, Qin L, Forgues M, et al. Predicting hepatitis B virus-positive metastatic hepatocellular carcinomas using gene expression profiling and supervised machine learning. Nat Med, 2003, 9: 416-423

21 Wei Y, Wang Q, Zhao X, et al. Immunotherapy of tumors with xenogeneic endothelial cells as a vaccine. Nat Med, 2000, 6: 11601166

22 Guo Y, Che X, Shen F, et al. Effective tumor vaccines generated by in vitro modification of tumor cells with cytokines and bispecific monoclonal antibodies. Nat Med, 1997, 3: 451-455

23 Zhang M, Tang H, Guo Z, et al. Splenic stroma drives mature dendritic cells to differentiate into regulatory dendritic cells. Nat Immunol, 2004, 5: 1124-1133

24 Zhu H, Zhou K, Jin C B, et al. High intensity focused ultrasound (HIFU) therapy for local treatment of hepatocellular carcinoma: Role of partial rib resection. Eur J Radiol, 2009, 72: 160-166

Open Access This article is distributed under the terms of the Creative Commons Attribution License which permits any use, distribution, and reproduction in any medium, provided the original author(s) and source are credited. 